\title{
On the evaluation of noise immunity of different classes of wideband signals
}

\author{
Evgeny G. Zhilyakov, Sergei P. Belov, Andrei S. Belov, Alexander S. Belov, Sergey A. Rachinsky
}

Belgorod State National Research University, Russia

\begin{tabular}{l}
\hline \hline Article Info \\
\hline Article history: \\
Received Aug 21, 2019 \\
Revised Nov 12, 2019 \\
Accepted Feb 24, 2020 \\
\hline
\end{tabular}

\section{Keywords:}

Broadband channel signals Interference

Signal bandwidth

Signal base

Wireless communication

systems

\begin{abstract}
The current stage of the development of the society is characterized by a continuous increase in remote interaction between subscribers and user requests for various types of multiservice services, with the required quality regardless of their location. This is mainly implemented on the basis of wireless communication systems (SBS) at the current time. A large number of various SBSs existing at the present leads to an increase in the level of various types of interference, which makes it necessary to increase the noise immunity of these systems. To improve the reliability of receiving information in the conditions of various types of interference, as is known, it is necessary to implement the information exchange by using channel signals with a large base, which primarily depends on the bandwidth they occupy. In this regard, in the present article, a comparative assessment of the noise immunity is carried out based on the obtained quantitative values of frequency characteristics of a number of modern classes of broadband channel signals, including a new class of broadband channel signals, and the use of eigenvectors of sub-band matrices.
\end{abstract}

This is an open access article under the CC BY-SA license.

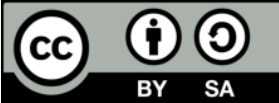

\section{Corresponding Author:}

Evgeny G. Zhilyakov,

Belgorod State National Research University,

85 Pobedy Street, Belgorod, the Belgorod region, 308015, Russia.

Email: zhilyakov@bsu.edu.ru

\section{INTRODUCTION}

The analysis of the existing approaches to the formation of broadband channel signals (PSS) [1-6] showed that the following methods are currently used for these purposes. The UWB technology, and in particular its impulse ra- dio (IR) version characterized by the transmission of a few nanoseconds duration pulses [7-12], offers an extraordinary resolution and localization precision in harsh environments, due to its ability to resolve multipath and penetrate obstacles [13-19]. Additional advantages include low power consump- tion, low probability of intercept, robustness to jamming, and coexistence with a large number of systems in the ever increasing spectrum exploitation [20-25].

\section{METHODOLOGY}

\subsection{Direct spread spectrum}

The essence of this method is as follows. Each element of the information sequence (" 1 " and " 0 ") is mapped to pseudo-random sequences (SRPs) different from each other, which then modulate in the phase of the high-frequency oscillation to obtain the SCNS. In the literature, such classes of signals are called t FM PSP signals. In mathematical form, FM PSP signals can be represented as follows: 


$$
S(t)=S_{0} \cdot \sum_{l=1}^{N} v_{l} \cdot \operatorname{rect}\left\{\frac{t-(l-1) \cdot \tau_{\ni}-\frac{T}{2}-\frac{\tau_{\ni}}{2}}{\tau_{\ni}}\right\}
$$

where $\tau_{\ni}$ is the length of the element of the SRP; $\mathrm{N}$ is the number of elements in the SRP; $v_{l}$ is the coefficient characterizing the state of the SRP takes values +1 or $-1, \operatorname{rect}(x)=1$, npu $|x| \leq \frac{1}{2} ; \operatorname{rect}(x)=0, \operatorname{npu}|x|>\frac{1}{2}$ -rectangular "cutting off" function. The type of SRP with the number of elements $\mathrm{N}=31$, obtained at the output of the register with feedbacks, which are calculated by a special algorithm using a computer, is shown in Figure 1.

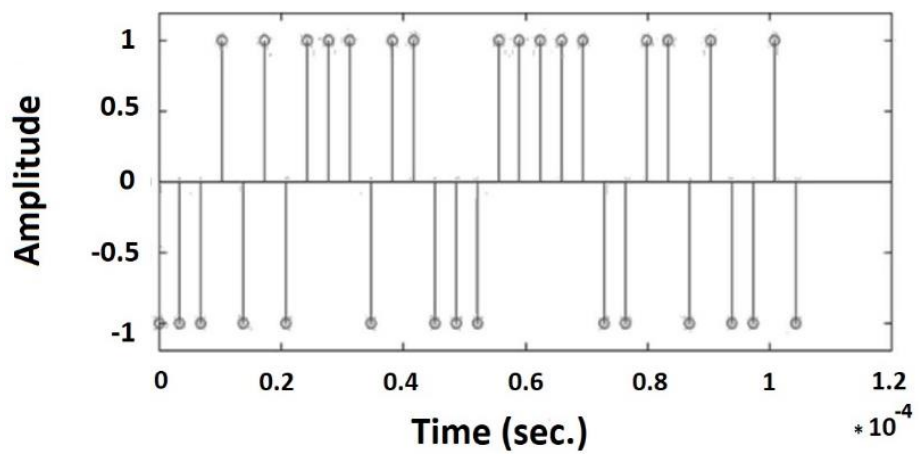

Figure 1. Type of pseudo-random sequence, (M-sequence) $\mathrm{N}=31$

\section{RESULTS}

\subsection{The method of forming pss using wavelet functions}

One of the promising areas $[7-9,17,22]$ that provides the formation of PSS with a bandwidth much higher than the width of the band provided by the direct spreading method, is the use of wavelet functions as the elements modulating the high-frequency oscillation. The analysis of a sufficiently large number of wavelet functions given in $[8,23]$ shows that the Morlet and Shannon wavelet functions are the most effective for the formation of the SCNS. Morlet's wavelet is asymmetric wavelet, and can be presented in the mathematical form as follows:

$$
\phi(x)=\exp \left(-\frac{x^{2}}{2}\right) \cdot \operatorname{Cos}(5 x)
$$

where $x$ is the values of current readings. The PLCS view obtained using the Morlet wavelet is shown in Figure 2.

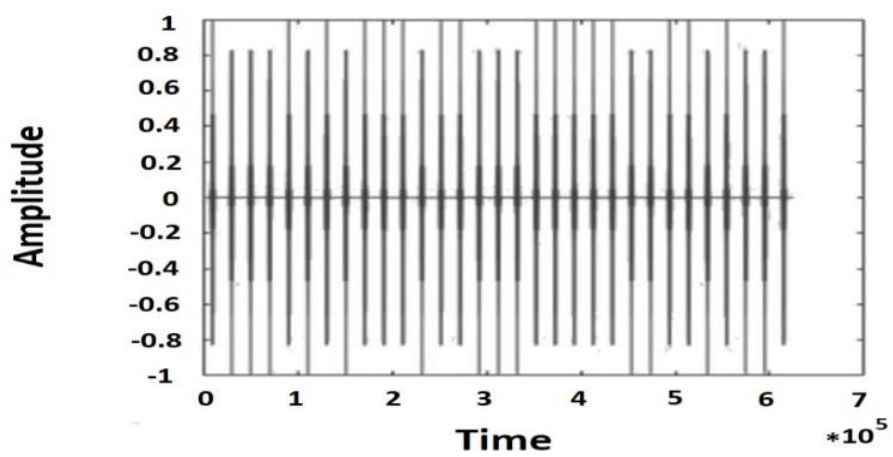

Figure 2. Type of PSS obtained using Morlet's wavelet 
It should be noted that Morlet's wavelet can be represented in a complex form using (3) [8]:

$$
\phi(x)=\frac{1}{\sqrt{\pi \cdot B}} \cdot \exp (j 2 \pi \cdot C \cdot x) \cdot \exp \left(-x^{2} / B\right)
$$

where $\mathrm{B}$ is a variable defining the bandwidth of frequencies, and $\mathrm{C}$ is a variable defining the center frequency. The type of PACS obtained by using the Morlet complex wavelet is shown in Figure 3.

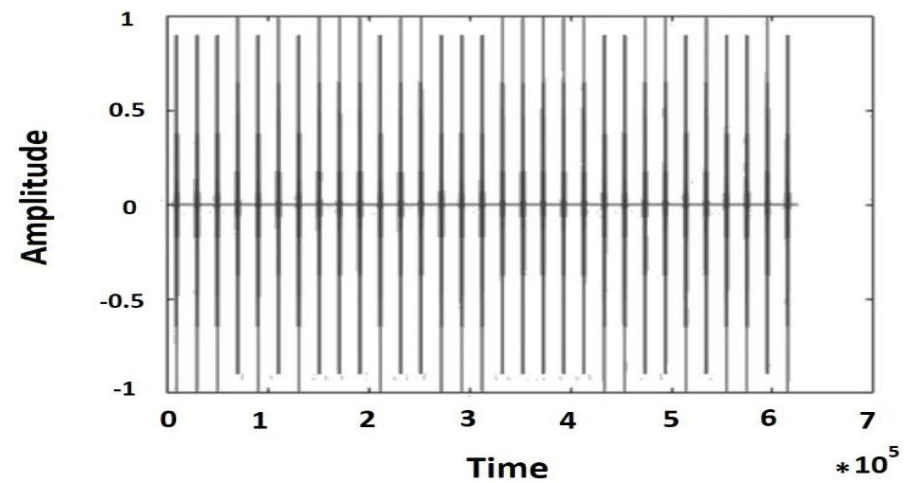

Figure 3. Type of PSSS obtained by using the Morlet complex wavelet

The complex Shannon wavelet in mathematical form can be represented as follows:

$$
\varphi(x)=\left(\sqrt{F_{b}}\right) \cdot\left[\operatorname{Sins}\left(F_{b}\right) \cdot \exp \left(2 j \pi F_{c} x\right)\right]
$$

where $F_{b}$ is the value of the frequency band of the wavelet function, is the value of the center frequency of the wavelet function, $\mathrm{x}$ is the value of the current samples, while $F_{c} \leq F_{b} / 2$. The type of PACS obtained using the Shannon wavelet is shown in Figure 4.

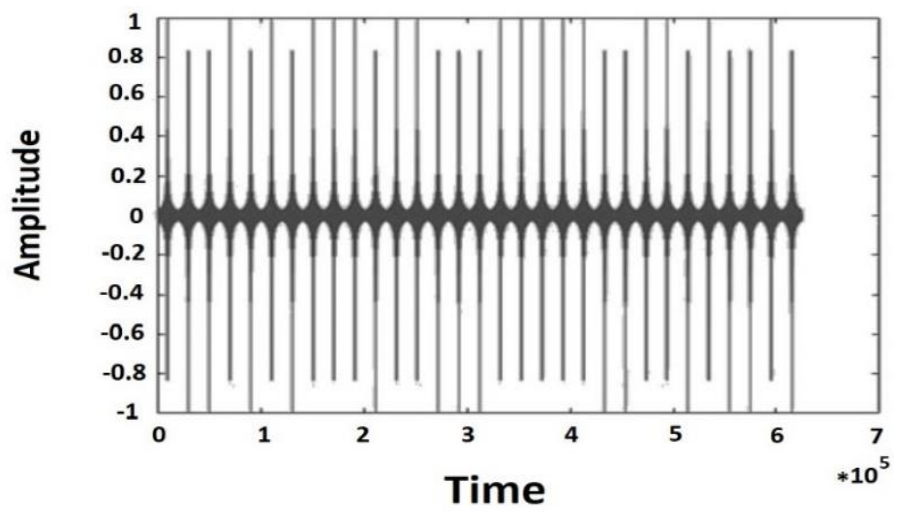

Figure 4. Kind of NLS obtained using a Shannon wavelet

\subsection{The method for formation of nics using the eigenvectors of sub-band matrices}

The method of generating PSS, using eigenvectors of sub-band matrices [10-14, 22, 24], is to use for transmission of information elements ("1" and "0") transmitting messages of different SRP, with each element of these sequences ( 1 and -1) proposed to transfer the opposite eigenvectors with eigenvalues close to unity, which are selected from the set of eigenvectors of the sub-band matrix with the elements of the form: 


$$
A_{i, j}= \begin{cases}\frac{\operatorname{Sin}(v(i-j))}{\pi(i-j)} & , i \neq j \\ v / \pi & i=j\end{cases}
$$

where the indices $i$ and $j$ take values with a step of 1 from 0 to $\mathrm{L}$ (the dimension of the matrix, i.e. the number of samples of the eigenvector), and vis the coefficient determining the bandwidth of the eigenvector being formed [15]. The PSCS view obtained using the eigenvectors of sub-band matrices is shown in Figure 5.

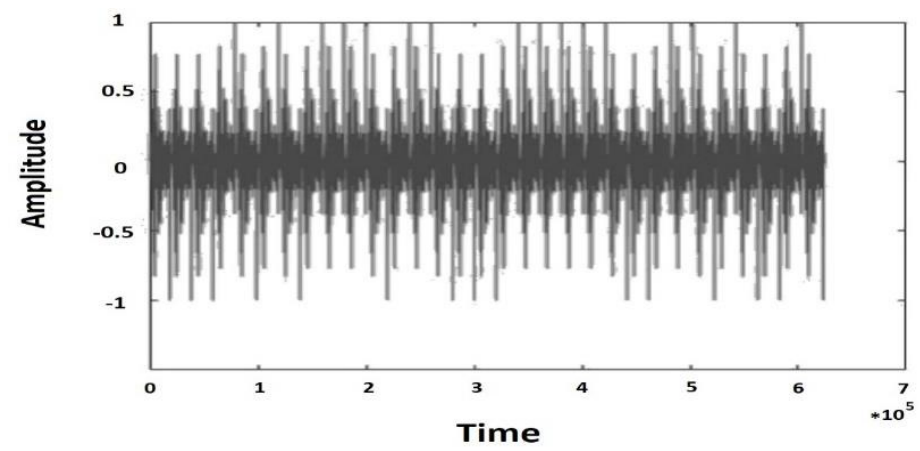

Figure 5. KINS type obtained using the eigenvectors of sub-band matrices

For a comparative analysis of the bandwidth that each of the studied signals occupies, the following parameters were used in the computational experiments [7]:

a. The information transfer rate $\mathrm{v}=9.6 * 103 \mathrm{kbit} / \mathrm{s}$;

b. The frequency of discredit $f_{d}=6 \mathrm{Ghz}$;

c. The carrier frequency $\mathrm{f}=1.646 \mathrm{Ghz}$;

d. The duration of the information sequence $\mathrm{T}=10^{-4}$ seconds

The results of computational experiments are presented in Figures 6 to 9, and they are summarized as well in Table 1. It should be noted that the probability of erroneous reception when using PACS in telecommunication systems is calculated by the following formula [16- 20, 22]:

$$
P_{\text {ош }}=0,5 \cdot\left(1-F\left(\sqrt{\frac{P_{\mathrm{c}}}{P_{\mathrm{m}}} \cdot \Delta F_{\mathrm{c}} \cdot T_{\mathrm{c}}}\right)\right)
$$

where $\Delta F_{c}$ is signal bandwidth, $T_{c}$ is the duration of PACS, $P_{c}$ is the PSS power in the signal frequency band $\Delta F_{c}, P_{u}$ is the noise power in the signal bandwidth $\Delta F_{c}, \mathrm{~F}(h)$ is the Crump function [21].

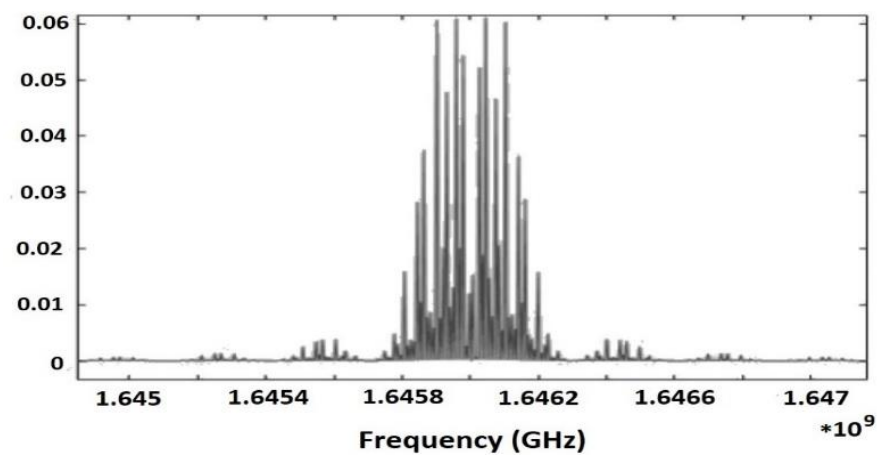

Figure 6. Frequency response of the FM PSP signal with the length of the PSP N=31 


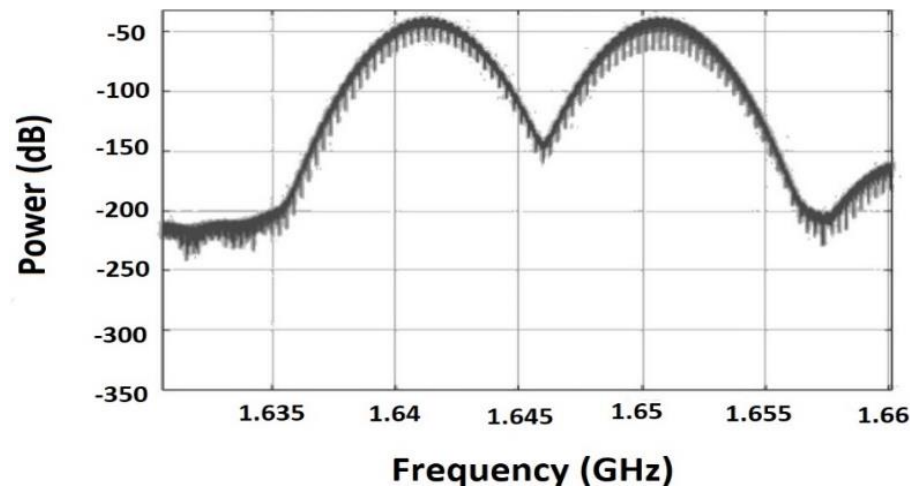

Figure 7. Frequency response of PSS using Morlet wavelet

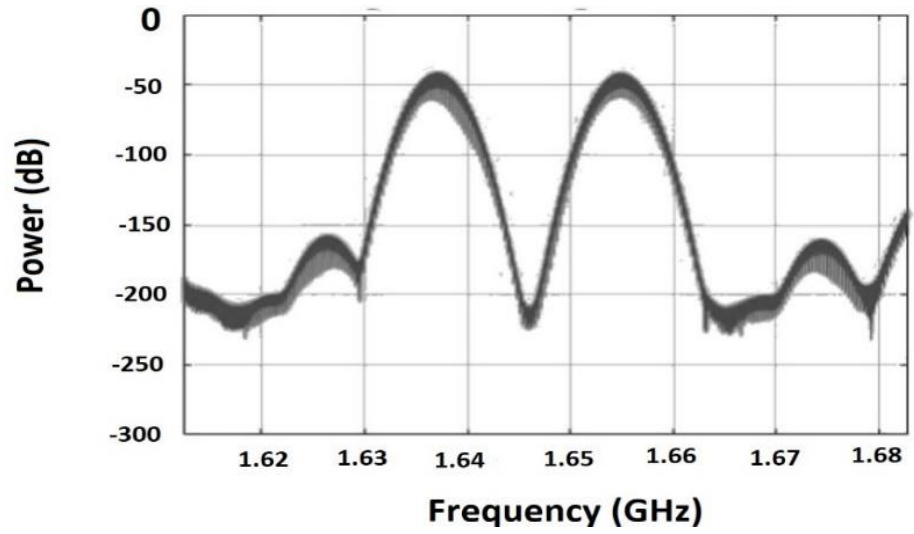

Figure 8. Frequency response of PSS using the Morlet complex wavelet

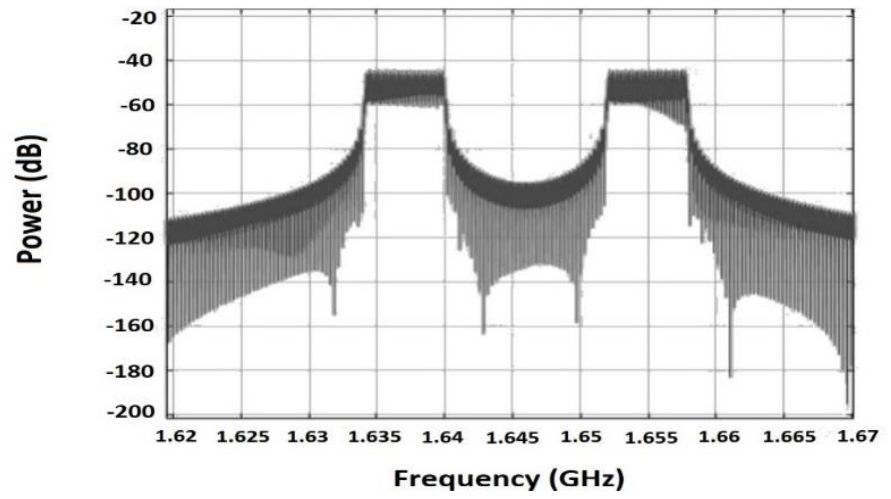

Figure 9. Frequency response of the SCNS using the Shannon wavelet

Table 1. Values of the bandwidth of the studied signals

\begin{tabular}{|c|c|c|c|c|c|c|}
\hline \multirow{2}{*}{ Type PAS } & \multicolumn{6}{|c|}{ PSP length, bit } \\
\hline & 31 & 63 & 127 & 511 & 1023 & 2047 \\
\hline FM PSP signals & 0.3 & 0.6 & 1.2 & 4.8 & 9.6 & 19.2 \\
\hline PSCS obtained using Morlet's wavelet & 20 & 42 & 84 & 336 & 672 & 1344 \\
\hline $\begin{array}{l}\text { PSTS obtained using the complex Morlet wavelet } \\
\mathrm{Fb}=1 ; \mathrm{Fc}=1.5 \text {. }\end{array}$ & 28 & 56 & 112 & 448 & 896 & 1792 \\
\hline $\begin{array}{l}\text { The PACS obtained using the Shannon wavelet } \\
\mathrm{Fb}=1 ; \mathrm{Fc}=1.5 \text {. }\end{array}$ & 24 & 48 & 96 & 387 & 768 & 1559 \\
\hline $\begin{array}{l}\text { NLS obtained using the eigenvectors of the } \\
\text { subband matrices } L=1024\end{array}$ & 48 & 100 & 200 & 800 & 1600 & 3200 \\
\hline
\end{tabular}

On the evaluation of noise immunity of different classes of wideband signals (Evgeny G. Zhilyakov) 


\section{CONCLUSION}

Undoubtedly today, communication and information exchange in its various forms plays a fundamental role in the creation and development of industrial infrastructure. Without the minimum of communication, one can never have proper management at the level of a large industrial project. It is important, of course, to maintain a reliable and uninterrupted communication in pursuit of the goals of the industrial complex. The technical nature of various communication systems, the relevance of communication equipment to other telecommunications networks, and the possibility of disconnection for a variety of reasons make us look for an idea that can provide a secure and stable connection that will meet the communication needs in all circumstances. This is mainly implemented on the basis of wireless communication systems (SBS) at the current time.

In the current study, it was tried to improve the reliability of receiving information in the conditions of various types of interference. For this reason, in the present paper, a comparative assessment of the noise immunity is carried out based on the obtained quantitative values of frequency characteristics of a number of modern classes of broadband channel signals, including a new class of broadband channel signals, and the use of eigenvectors of sub-band matrices. From the analysis of the data presented in Table 1, it can be seen that the broadband channel signals based on the use of the eigenvectors of sub-band matrices, have significantly greater bandwidth than other types of SCNRs whose properties were presented in this article. Consequently, their use in wireless communication systems will significantly improve the reliability of information exchange.

\section{ACKNOWLEDGMENTS}

The present work was done with the financial support of the Ministry of Education and Science in the framework of the state assignment of the Belarusian State University (Project \# 8.2201.2017/4.6).

\section{REFERENCES}

[1] D. V. Ivanov, et al., "Propagation of broadband HF signals in a medium with nonlinear dispersion," Journal of Communications Technology and Electronics, vol. 60, no. 11, pp. 1205-1214, 2015.

[2] P. K. Dickson, "Broadband systems [Shirokopolosnyye sistemy],"Transl. from English M.: Communication, 1979.

[3] C. Cook, "Radar signals: An introduction to theory and application," Academic Press, 1967.

[4] W. C. Hoffman, "Statistical methods in radio wave propagation," Proceedings of a symposium held at the University of California, Pergamon Press, 1960.

[5] A. Serkov, et al., "Noise-like signals in wireless information transmission systems," Advanced Information Systems, vol. 1, pp. 33-38, 2017.

[6] G. I. Tuzov, "Noise immunity of radio systems with complex signals," Moscow Izdatel Radio Sviaz, p. $264,1985$.

[7] E. Sharghi, et al., "Emotional ANN (EANN) and wavelet-ANN (WANN) approaches for Markovian and seasonal based modeling of rainfall-runoff process,"Water resources management, vol. 32, no. 10, pp. 3441-3456, 2018.

[8] N. K. Smolentsev, "Fundamentals of the theory of wavelets,"Wavelets in MATLAB-Moscow, vol. 304. 2005.

[9] E. Sharghi, et al., "Conjunction of emotional ANN (EANN) and wavelet transform for rainfall-runoff modeling," Journal of Hydroinformatics, vol. 21, no. 1, pp. 136-152, 2019.

[10] E. G. Zhilyakov, et al., "Variational methods of synthesis of signals based on the frequency of ideas,"Journal of Theoretical and Applied Information Technology, vol. 92, no. 1, pp. 59-63, 2016.

[11] G. Ungerboeck, "Channel coding with multilevel/phase signals,"IEEE transactions on Information Theory, vol. 28, no. 1 , pp. 55-67, 1982.

[12] E. G. Zhilyakov, et al., "Image decomposition on the orthogonal basis of subband matrics eigenvectors,"Journal of Engineering and Applied Sciences, vol. 12, no. 12, pp. 3194-3197, 2017.

[13] H. Nikookar and R. Prasad, "Optimal waveform design for multicarrier transmission through a multipath channel," IEEE 47th Vehicular Technology Conference. Technology in Motion, vol. 3, pp. 1812-1816, 1997.

[14] H. Erdogan, et al., "Multi-channel speech recognition: LSTMs all the way through," CHiME-4 workshop, pp. 1-4, 2016

[15] O. Martens, et al., "Phase-increment sampling in chirp signal based impedance measurements," International Conference on Sampling Theory and Applications (SampTA), pp. 604-608, 2017.

[16] H. Yang, "The novel modern internet of things system structure optimization methodology based on information theory and communication signal transmission model,"International Journal of Future Generation Communication and Networking, vol. 9, no. 9, pp. 119-132, 2016.

[17] V. Nourani, et al., "Hybrid wavelet-M5 model tree for rainfall-runoff modeling,"Journal of Hydrologic Engineering, vol. 24 , no. $5,2019$.

[18] V. O. Kalinin and V. I. Nosov, "Assessment of effect of influences on pulse-based UWB radio communication systems," International Siberian Conference on Control and Communications (SIBCON), pp. 1-6, 2016.

[19] L. E. Varakin, “Theory of signal systems,"Moscow, Izdatel'stvo Sovetskoe Radio, p. 304, 1978. 
[20] K. A. Lukin and O. V. Zemlyaniy, "Digital generation of wideband chaotic signal with the comb-shaped spectrum for communication systems based on spectral manipulation," Radioelectronics and Communications Systems, vol. 59, pp. 417-422, 2016.

[21] M. Soleimani and Z. M. Beigi, "Effective indicators in bank customer satisfaction," UCT Journal of Social Sciences and Humanities Research, vol. 2, no. 1, pp. 41-45, 2014.

[22] V. Nourani, et al., "Data mining based on wavelet and decision tree for rainfall-runoff simulation," Hydrology Research, vol. 50, no. 1, pp. 75-84, 2019.

[23] M. Pradeep, et al., "Dynamic smart alert service for women safety system," International Journal of Communication and Computer Technologies, vol. 5, no. 2, pp. 58-66, 2017.

[24] S. K. Hildayanti and J. Alie, "Factors influenced paddy farmers to use or not use organic fertilizers in South Sumatera, Indonesia," Humanities \& Social Sciences Reviews, vol. 4, no. 1, pp. 53-58, 2016.

[25] M. R. Mosavi, et al., "A fast and accurate anti-jamming system based on wavelet packet transform for GPS receivers,"GPS solutions, vol. 21, no. 2, pp. 415-426, 2017.

\section{BIOGRAPHIES OF AUTHORS}

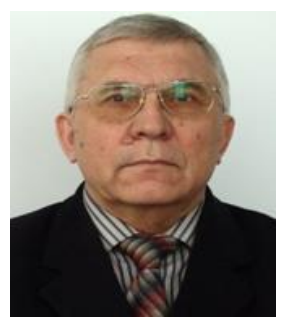

Evgeny G. Zhilyakov received the degree of Doctor of Technical Sciences in 1994. In 1997 he was awarded the academic title of professor. He is currently head of the Department of Belgorod State National Research University. His research interests include signal analysis and synthesis theory, methods for minimizing the costs of time-frequency resources of information transmission channels.

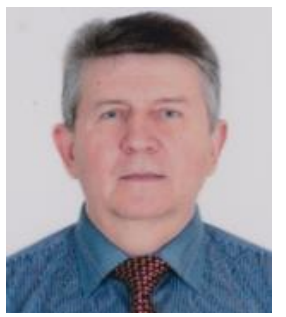

Sergei P. Belov received a doctorate in technical sciences in 2012. In 2015, he was awarded the academic title of professor. Currently he is a professor at the Department of Information and Telecommunication Systems and Technologies, Belgorod State National Research University. His research interests include the theory of the formation and processing of channel signals, methods of minimizing the costs of time-frequency resources of information transmission channels.

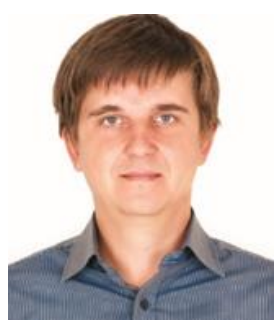

Alexander S. Belov received his Ph.D. degree in technical sciences in 2009. In 2015, he received the academic title of Associate Professor. He is currently an assistant professor at the Department of Information and Telecommunication Systems and Technologies, Belgorod State National Research University. His research interests include methods for improving the reliability of information transfer in infocommunication systems.

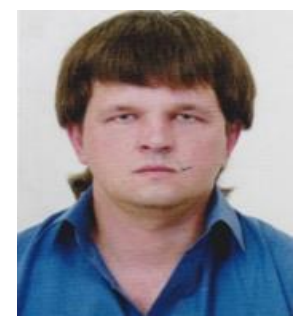

Andrei S. Belov has a specialist degree in communications systems and management, and is currently studying for graduate studies at Belgorod State National Research University. The topic of his thesis is the improvement of methods and algorithms for transmitting information in satellite communication systems. His research interests include channel signal generation and processing.

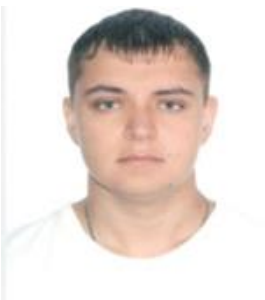

Sergey A. Rachinsky received a degree in communications networks and switching systems at the Belgorod State National Research University in 2015. In 2019 he received the degree of candidate of technical sciences. He is an assistant at the Department of Information and Telecommunication Systems and Technologies. His research interests include channel signal generation and processing. 